\title{
Review Article \\ Systematic Review of Multidisciplinary Chronic Pain Treatment Facilities
}

\author{
Samantha R. Fashler, ${ }^{1,2}$ Lynn K. Cooper, ${ }^{2}$ Eric D. Oosenbrug, ${ }^{1}$ Lindsay C. Burns, ${ }^{1}$ \\ Shima Razavi, ${ }^{1}$ Lauren Goldberg, ${ }^{1}$ and Joel Katz ${ }^{1,2}$ \\ ${ }^{1}$ Department of Psychology, York University, Toronto, ON, Canada M3J 1P3 \\ ${ }^{2}$ Canadian Pain Coalition, Oshawa, ON, Canada L1J 8P7
}

Correspondence should be addressed to Lynn K. Cooper; lkcooperbes@rogers.com and Joel Katz; jkatz@yorku.ca

Received 4 December 2015; Accepted 29 December 2015

\begin{abstract}
Copyright ( 2016 Samantha R. Fashler et al. This is an open access article distributed under the Creative Commons Attribution License, which permits unrestricted use, distribution, and reproduction in any medium, provided the original work is properly cited.

This study reviewed the published literature evaluating multidisciplinary chronic pain treatment facilities to provide an overview of their availability, caseload, wait times, and facility characteristics. A systematic literature review was conducted using PRISMA guidelines following a search of MEDLINE, PsycINFO, and CINAHL databases. Inclusion criteria stipulated that studies be original research, survey more than one pain treatment facility directly, and describe a range of available treatments. Fourteen articles satisfied inclusion criteria. Results showed little consistency in the research design used to describe pain treatment facilities. Availability of pain treatment facilities was scarce and the reported caseloads and wait times were generally high. A wide range of medical, physical, and psychological pain treatments were available. Most studies reported findings on the percentage of practitioners in different health care professions employed. Future studies should consider using more comprehensive search strategies to survey facilities, improving clarity on what is considered to be a pain treatment facility, and reporting on a consistent set of variables to provide a clear summary of the status of pain treatment facilities. This review highlights important information for policymakers on the scope, demand, and accessibility of pain treatment facilities.
\end{abstract}

\section{Introduction}

Chronic pain is a major global health problem that affects approximately $37 \%$ of individuals in developed countries [1]. In Canada, it is estimated that as many as $25 \%$ of adults have a chronic pain condition [2]. Chronic pain places a substantial burden on the individual and can contribute to disability [3], depression [4], anxiety [5], and lowered quality of life [6]. There are also significant societal costs associated with chronic pain. In the United States, chronic pain is estimated to cost between 560 and 635 billion dollars annually [7], while, in Canada, direct costs to the health care system are estimated to be over 6 billion dollars and indirect costs due to job loss and sick days are estimated to be over 37 billion dollars annually $[8,9]$.

The development and maintenance of chronic pain is complex, affected by biological, psychological, and social factors $[5,10]$. Consequently, the most effective pain treatment strategies target a variety of factors simultaneously, an approach that is used in multidisciplinary pain treatment facilities [11-13]. The services and standards of multidisciplinary treatment vary considerably, which led the International Association for the Study of Pain (IASP) to release guidelines regarding the classification and ideal standards of pain services [14]. IASP defines a "multidisciplinary pain center" (a Level 1 facility) as being staffed by a variety of health care professions with expertise in pain management, including physicians, nurses, mental health professionals, and physical therapists. The team should work together and be able to effectively assess and treat any pain problem. Training opportunities, education, and research should be available at the pain center. A "multidisciplinary pain clinic" (a Level 2 facility) must uphold the same standards of a multidisciplinary pain center, although they may not have training and research opportunities. Further classification includes "pain syndrome programs" that provide multidisciplinary care for one type of pain and "single modality therapy programs" that provide one type of treatment. A "pain treatment facility" is a general term that encompasses all of the above definitions [15]. 
Despite being considered the highest standard of care for chronic pain, there is limited research regarding the availability and characteristics of pain treatment facilities globally. In order to provide a comprehensive overview of the published literature, the Canadian Pain Coalition (http://www.canadianpaincoalition.ca/) sponsored the present systematic review as the first phase of a "Report Card on Pain." This initiative aims to identify areas of strength and weakness in pain treatment facilities worldwide to help inform policymakers on targeted funding strategies to better tackle the challenge of pain in Canada and globally. The objective of the present paper is to systematically review the published literature of surveys on multidisciplinary pain treatment facilities to provide an overview of their availability, caseload, and wait times, as well as the facility characteristics, including available treatments and employed pain professionals.

\section{Methods}

2.1. Search Strategy. The systematic review was conducted according to PRISMA guidelines [16]. The review protocol was developed by the authors prior to beginning the searches. Eligibility criteria required articles to meet four study characteristics: (1) studies must describe pain treatment facilities directly with questionnaires or other data collection methods (excluding qualitative studies); (2) studies must be original research articles (i.e., no editorials, reviews); (3) more than one multidisciplinary pain treatment facility must be surveyed; and (4) questionnaires must describe a range of pain treatments rather than focusing on one type or category of treatment (e.g., acupuncture). At this stage, no restrictions were placed on report characteristics (e.g., language), publication status, location of study, or date of publication. Additionally, studies were not required to be published by an academic journal and could be published by an established organization.

The search strategy included searching academic article databases, asking experts in the field, and reviewing reference lists of included articles. No language restrictions were made for the initial search. Database searches were conducted using MEDLINE (1946 to August 2014), PsycINFO (1912 to August 2014), and CINAHL (1979 to August 2014). The last search was completed on September 23, 2014. All authors developed the search protocol and one author (Joel Katz) conducted the searches. The authors of all included articles were contacted for the original questionnaire used in their study.

The following search terms were used: pain; pain clinic*; pain service*; pain cent ${ }^{*}$; pain facilit*; pain management clinic $^{*}$; pain management service*; pain management cent ${ }^{*}$; pain management facilit*; pain treatment clinic ${ }^{*}$; pain treatment service*; pain treatment cent ${ }^{*}$; pain treatment facilit ${ }^{*}$; survey $^{*}$; program evaluation; program assessment; program outcome; questionnaire* . See Appendices A and B for a complete description of the search strategy and search terms.

2.2. Data Collection. Study titles and abstracts were reviewed for inclusion by one author (Joel Katz) according to the eligibility criteria. Articles selected for full-text review were independently reviewed by two authors (Joel Katz and Samantha R. Fashler). Disagreements were discussed until a consensus could be reached.

Data was transposed into a data extraction spreadsheet collaboratively created by three authors. The spreadsheet was modified as needed to accommodate new information from studies as they were reviewed. One author entered the extracted data from the studies into the spreadsheet (Samantha R. Fashler) and one author checked the data that was entered (Eric D. Oosenbrug). Study authors were contacted to receive a copy of the survey used in the project. Six authors provided a copy of the survey, one author responded to say they did not have a copy of the survey, five authors did not respond, and one survey was available in the appendix of the published study. In one case, results on the same survey were reported in two studies $[17,18]$. To avoid bias, no duplicate information was extracted and nonoverlapping content was included in the extraction spreadsheet.

All variables included in the data extraction spreadsheet related to the assessment and evaluation of pain treatment facilities. Specifically, information related to following domains was extracted: (1) study characteristics, including a definition of pain treatment facilities used, search strategy, location and date surveyed, questionnaire used, and the response rate; (2) access to pain treatment facilities, including the rate of new and follow-up consultations and wait times; (3) pain treatments available, including medical approaches, physical therapies, and psychological services; and (4) characteristics of pain treatment facilities, including the employed health care professionals, staff, space, and equipment.

Risk of bias was assessed with a tool developed by the Cochrane Collaboration [19]. It is a domain-based evaluation tool, assessing the following: selection bias, performance bias, detection bias, attrition bias, reporting bias, and other biases (not addressed with the former biases).

Summary measures included standardized differences in means. Data transformation was used to increase comparability across reported data: specific alterations to original data are described in their respective table. No additional analyses were undertaken.

\section{Results}

3.1. Study Selection. Searches conducted in MEDLINE, PsycINFO, and CINAHL yielded 2117 hits. Eighteen additional articles were hand selected by consulting with experts and searching reference lists. After duplicates were removed, 1692 unique articles remained. Articles were first screened by title and abstract by one author (Joel Katz), eliminating 1627 articles. The full texts of the remaining 65 articles were reviewed by two authors (Joel Katz and Samantha R. Fashler). Fifty-one articles were eliminated because they did not investigate pain treatment facilities $(n=26)$ or because they did not meet the selection criteria: eight articles surveyed clinicians or patients directly rather than pain treatment facilities, three only looked at one pain treatment facility, and three articles only examined one type of service. The original 


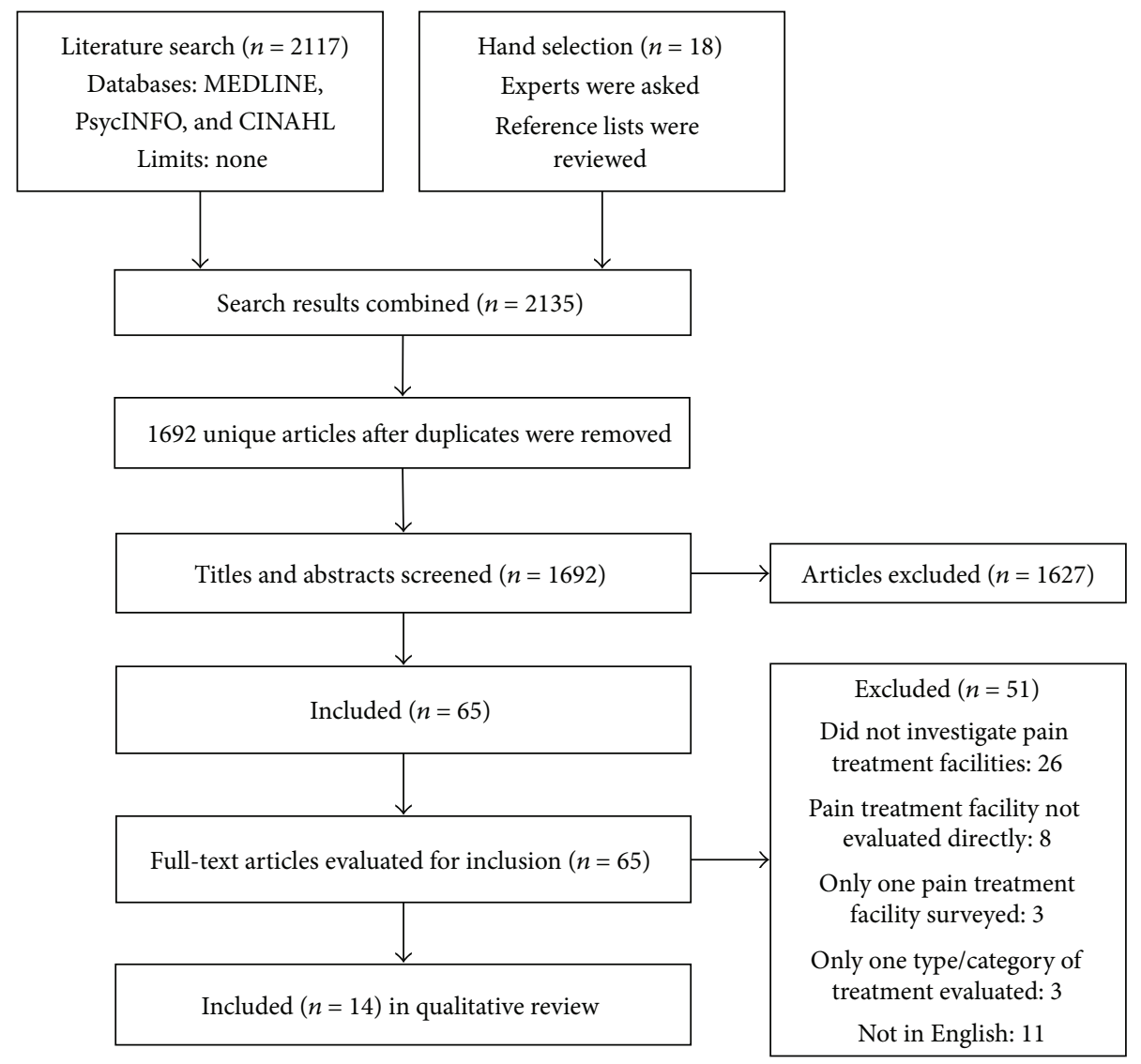

Figure 1: Flow diagram of study selection.

study design aimed to include non-English articles. However, the 11 non-English articles identified were eliminated from the present review due to difficulties in accurately translating them. See Figure 1 for a flow diagram of the articles selection and inclusion process used. In total, 14 studies were included in the present review.

Reporting bias may be present in the studies included in the review. When the questionnaires were available, the reported outcomes in the paper were compared to the questionnaire used to determine if any relevant data were not reported. The studies included in the review had a recruitment bias, since many pain treatment facilities that were contacted did not respond to the surveys, and are therefore not represented in the reported survey findings. A selection bias was present for the baseline characteristics of the surveys as the definition of a pain treatment facility was different for each study.

3.2. Study Characteristics. The 14 included studies were published between 1985 and 2013 [17, 18, 20-31]. Surveys were carried out around the world, with five in the United Kingdom, four in Canada, three in the United States, one in Australia, and one in Italy. Surveys reported countrywide or region-specific data. Ten were published in scientific journals $[17,18,20-24,29-31]$, two were audits of pain services $[27,28]$, and two were reports published by professional organizations $[25,26]$. Despite examining the same topic, each study used different search criteria to find pain treatment facilities and used different eligibility criteria for what was considered a pain treatment facility (see Table 1 ). The response rate in studies ranged from $56 \%$ to $100 \%(\mathrm{M}=80.1 \%$; $95 \% \mathrm{CI}$ : 70.92 to 89.31 ). Each study has a bias according to the time it was conducted, location of the survey, the manner in which it was published (i.e., in a peer-reviewed journal or an independently published report or project), pain treatment facility search strategy, eligibility criteria, and response rate.

All studies used a survey that was sent by phone, fax, email, or mail. Only one study [20] included an interview as a part of data collection. Pain treatment facility directors, chief executive officers, head anesthesiologists, and, in one case, senior psychologists were asked to complete the questionnaire. Surveys were developed for each study with two exceptions: Peng et al. [17, 18, 21] used an adapted version of the Québec Chronic Pain Clinic Survey developed by Veillette et al. [22] and Csordas and Clark [30] did not specify what survey was used.

Health care systems can vary substantially depending on the country of origin [32]. For this reason, the results on pain facility characteristics are reported by country (Australia, Canada, Italy, United Kingdom, and United States).

3.3. Availability and Caseloads of Pain Treatment Facilities. Data on the availability, caseloads, and wait times for pain treatment facilities are listed in Tables 2 and 3. In many 


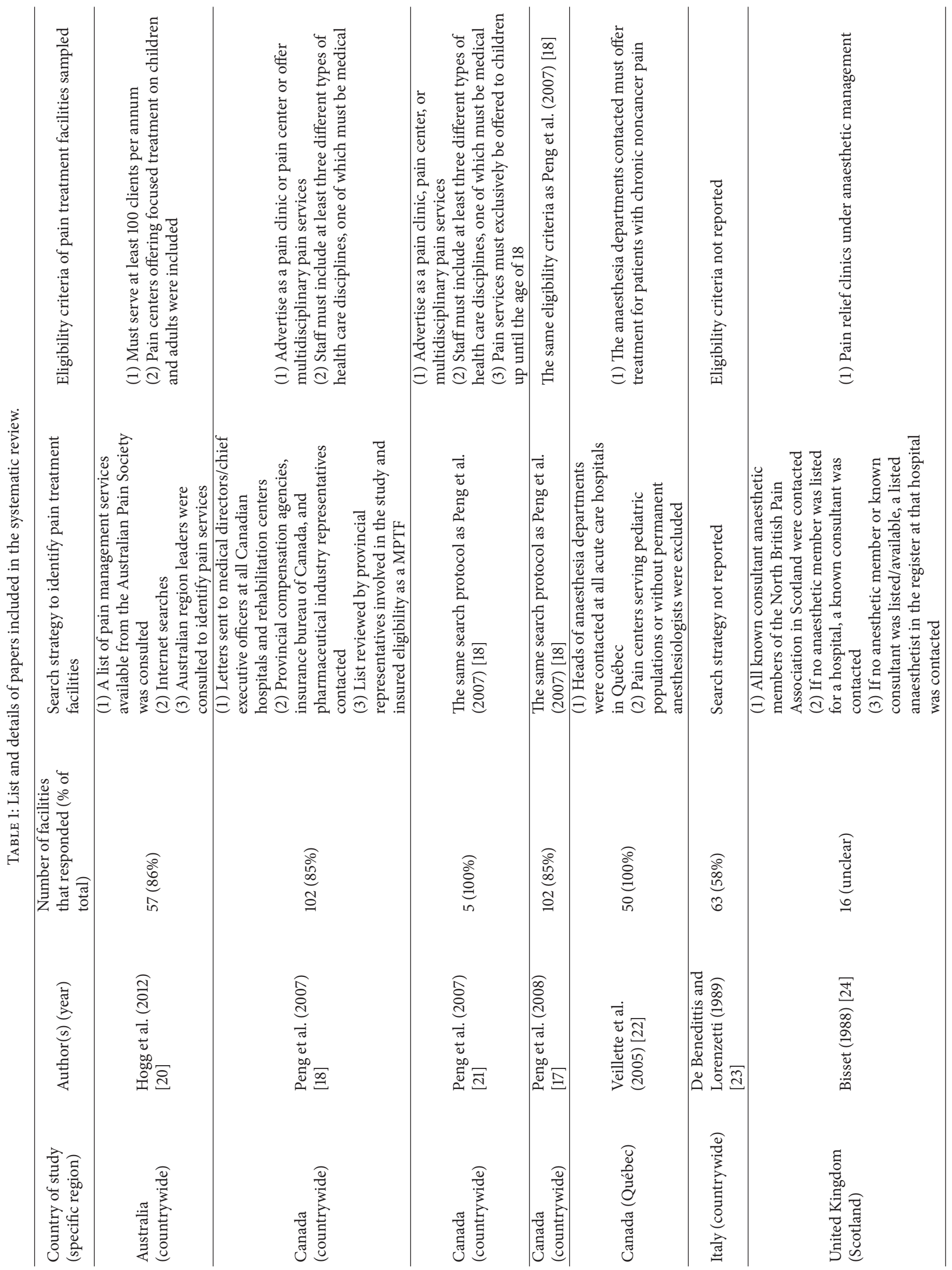




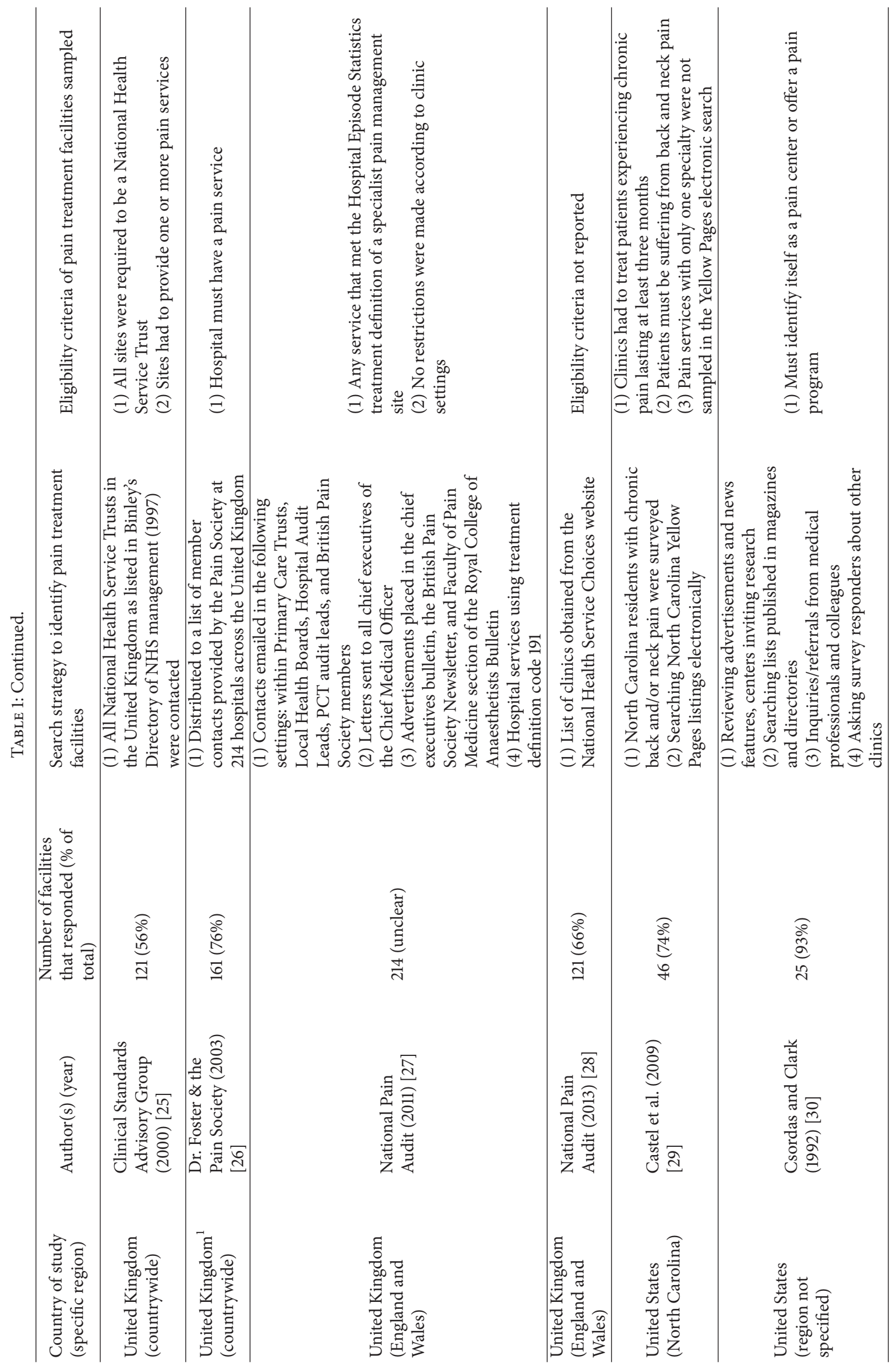




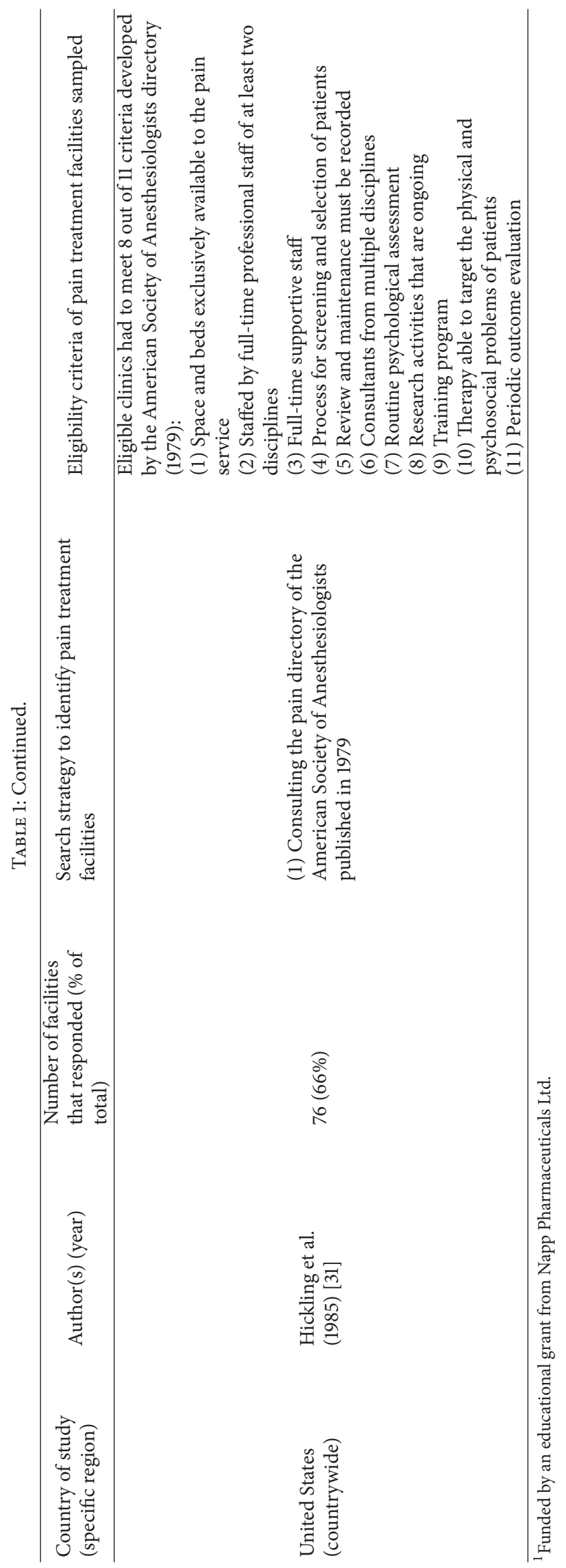


TABLE 2: Availability and caseload of pain treatment facilities.

\begin{tabular}{|c|c|c|c|c|}
\hline Author(s) (year) & $\begin{array}{c}\text { Pain facility per } \\
\text { population }\end{array}$ & $\begin{array}{l}\text { New consultation } \\
\text { appointments per } \\
\text { facility per month }\end{array}$ & $\begin{array}{l}\text { Follow-up } \\
\text { appointments per } \\
\text { facility per month }\end{array}$ & $\begin{array}{l}\text { Appointments } \\
\text { per facility per } \\
\text { month }\end{array}$ \\
\hline \multicolumn{5}{|l|}{ Australia } \\
\hline Hogg et al. (2012) [20] & $\begin{array}{l}1 \text { per } 310,000 \\
\text { people }\end{array}$ & $\begin{array}{c}\text { Range }= \\
24.92-36.75^{\mathrm{a}}\end{array}$ & - & - \\
\hline \multicolumn{5}{|l|}{ Canada } \\
\hline Peng et al. (2007) [21] & - & $\operatorname{Mdn}=2.58^{\mathrm{a}}$ & $\mathrm{Mdn}=37.50^{\mathrm{a}}$ & - \\
\hline Peng et al. (2007) [18] & $\begin{array}{l}1 \text { per } 258,000 \\
\text { people }\end{array}$ & $\mathrm{M}=37.58^{\mathrm{ab}}$ & $\mathrm{M}=432.19^{\mathrm{ab}}$ & - \\
\hline Veillette et al. (2005) [22] & - & $\mathrm{M}=16^{\mathrm{b}}$ & $\mathrm{M}=74^{\mathrm{b}}$ & - \\
\hline \multicolumn{5}{|l|}{ Italy } \\
\hline De Benedittis and Lorenzetti (1989) [23] & - & - & - & $\mathrm{M}=164.58^{\mathrm{a}}$ \\
\hline \multicolumn{5}{|l|}{ United Kingdom } \\
\hline Clinical Standards Advisory Group (2000) [25] & - & Average $=35$ & Average $=21$ & - \\
\hline Dr. Foster \& the Pain Society (2003) [26] & - & - & - & Range $=15-750^{\mathrm{a}}$ \\
\hline National Pain Audit (2011) [27] & $\begin{array}{l}\text { Range }=0.27-0.50 \\
\text { per } 100,000 \text { people }\end{array}$ & - & - & - \\
\hline \multicolumn{5}{|l|}{ United States } \\
\hline Castel et al. (2009) [29] & - & - & - & $\begin{array}{c}\mathrm{M}=1244.10^{\mathrm{c}} \\
\mathrm{Mdn}=709.05^{\mathrm{c}}\end{array}$ \\
\hline Hickling et al. (1985) [31] & - & $M=36.1$ & $M=74.3$ & - \\
\hline
\end{tabular}

Note. Information on incidence and caseload was not available for Bisset [24], Csordas and Clark [30], National Pain Audit [28], and Peng et al. [17].

${ }^{a}$ Divided by 12 to provide a monthly estimate since annual appointments were reported in source paper.

${ }^{b}$ Divided by the number of pain treatment services surveyed since the total number of appointments was reported in the source paper.

${ }^{\mathrm{c}}$ Multiplied by 4.35 to provide a monthly estimate since weekly appointments were reported in source paper.

cases, the metric used to report this information varied, with some studies reporting medians, means, ranges, or sums. To facilitate meaningful comparisons across studies, relevant data were transformed to match the metrics reported in other studies (e.g., if new consultation appointments were reported annually, it was divided by 12 to permit comparison to other articles that reported this index according to new consultations per month).

Australia. In their survey, Hogg et al. [20] found that there was only one pain treatment facility for 310,000 Australians. The authors reported that new consultation appointments ranged from 24.92 to 36.75 per month. The median wait time for public facilities was reported to be 150 days, with the median wait time of private facilities being 38.5 days. The reported median for all facilities was 103 days.

Canada. Only Peng et al. [18] reported on the proportion of pain facilities for the population, finding that there is one pain treatment facility for 258,000 people in Canada. They found that the average number of monthly consultations and wait times varied according to the type of facility surveyed. In pediatric pain treatment facilities [21], fewer new consultations $(\mathrm{Mdn}=2.58)$ and follow-up appointments $(\mathrm{Mdn}=37.50)$ were reported and the median wait time for all facilities was 28 days, with a median of 10 patients waiting for treatment. More appointments (new: $\mathrm{M}=37.58$, follow-up: $M=432.19$ ) were reported for adult pain treatment facilities
[18], with the median wait time reported being 180 days for public facilities and 15 days for private facilities. In hospital anesthesia departments in Québec [22], the reported caseload was $\mathrm{M}=16$ for new consultations and $\mathrm{M}=74$ for follow-up appointments, with 4500 patients awaiting treatment.

Italy. Only one measure of caseload was available for Italy. De Benedittis and Lorenzetti [23] reported $\mathrm{M}=164.58$ appointments per month for the 63 pain treatment facilities surveyed. No information was available regarding access to care or wait times.

United Kingdom. The National Pain Audit [27] estimated that there was one pain treatment facility for 200,000370,370 people living in the United Kingdom. The number of appointments varied [new: average $=35$, follow-up: average $=21$ [25]]. The Clinical Standards Advisory Group [25] reported wait times according to the type of care needed. The longest median wait time was reported for routine care (112 days), while the median wait time for urgent care was 14 days and only 7 days for cancer care. The report by Dr. Foster \& the Pain Society [26] indicated that the median wait times were longer for patients referred by consultants (161 days) in comparison to general practitioners (140 days). The National Pain Audit [27] reported that wait times for $80 \%$ of English facilities were less than 126 days, although only $50 \%$ of Welsh pain facilities had wait times meeting these criteria. 
TABLE 3: Pain treatment facility wait times and number of patients waiting for treatment.

\begin{tabular}{|c|c|c|c|c|}
\hline Author(s) (year) & $\begin{array}{l}\text { Public facilities, } \\
\text { median } \\
\text { (interquartile } \\
\text { range) }\end{array}$ & $\begin{array}{l}\text { Private facilities, } \\
\text { median } \\
\text { (interquartile } \\
\text { range) }\end{array}$ & $\begin{array}{l}\text { All facilities, } \\
\text { median } \\
\text { (interquartile } \\
\text { range) }\end{array}$ & $\begin{array}{c}\text { Number of } \\
\text { patients, median } \\
\text { (interquartile } \\
\text { range) }\end{array}$ \\
\hline \multicolumn{5}{|l|}{ Australia } \\
\hline Hogg et al. (2012) [20] & 150 days $(68-281)^{\mathrm{a}}$ & 38.5 days $(24-75)^{\mathrm{a}}$ & 103 days $(44-210)^{\mathrm{a}}$ & - \\
\hline \multicolumn{5}{|l|}{ Canada } \\
\hline Peng et al. (2007) [18] & 180 days $(60-420)^{\mathrm{b}}$ & 15 days $(9-30)^{\mathrm{b}}$ & - & - \\
\hline Peng et al. (2007) [21] & - & - & 28 days $(14-42)^{\mathrm{a}, \mathrm{b}}$ & $\begin{array}{l}10 \text { patients } \\
(2-17)\end{array}$ \\
\hline Veillette et al. (2005) [22] & - & - & - & $\begin{array}{l}4500 \text { patients, } \\
\text { totall }^{c}\end{array}$ \\
\hline United Kingdom & - & - & & \\
\hline $\begin{array}{l}\text { Clinical Standards Advisory } \\
\text { Group (2000) [25] }\end{array}$ & - & - & - & $\begin{array}{l}90 \text { patients } \\
(45-150)^{\mathrm{d}}\end{array}$ \\
\hline Routine care & - & - & 112 days $(70-196)^{\mathrm{b}, \mathrm{e}}$ & - \\
\hline Urgent care & - & - & 14 days $(7-14)^{\mathrm{b}, \mathrm{e}}$ & - \\
\hline Cancer care & - & - & $\begin{array}{c}7 \text { days }(7-14 \\
\text { days })^{\mathrm{b}, \mathrm{e}}\end{array}$ & - \\
\hline $\begin{array}{l}\text { Dr. Foster \& the Pain Society } \\
(2003)[26]\end{array}$ & - & - & - & - \\
\hline $\begin{array}{l}\text { Referred by general } \\
\text { practitioners }\end{array}$ & - & - & $\begin{array}{l}140 \text { days (total } \\
\text { range: } 28-770)^{\mathrm{b}}\end{array}$ & - \\
\hline Referred by consultants & - & - & $\begin{array}{l}161 \text { days (total } \\
\text { range: } 28-931)^{\mathrm{a}}\end{array}$ & - \\
\hline National Pain Audit (2011) [27] & - & - & - & - \\
\hline England & - & - & $\begin{array}{c}80 \% \text { of services } \\
\text { under } 126 \text { days }^{\mathrm{b}} . \text { If } \\
\text { above, } \mathrm{Mdn}=140 \\
\text { days }^{\mathrm{b}}\end{array}$ & - \\
\hline Wales & - & - & $\begin{array}{c}50 \% \text { of services } \\
\text { under } 126 \text { days }{ }^{\mathrm{b}} . \text { If } \\
\text { above, } \text { Mdn }^{\text {abys }}=231 \\
\text { days }^{\mathrm{b}}\end{array}$ & - \\
\hline
\end{tabular}

Note. Information on wait times and patients waiting for treatment was not available for Bisset [24], Castel et al. [29], Csordas and Clark [30], De Benedittis and Lorenzetti [23], Hickling et al. [31], National Pain Audit [28], and Peng et al. [17].

${ }^{a}$ Initial/first assessments.

${ }^{\mathrm{b}}$ Converted from weeks or months to days.

${ }^{\mathrm{c}}$ Of this group, $67 \%$ were waiting for longer than 9 months.

${ }^{\mathrm{d}}$ New and old patients.

${ }^{\mathrm{e}} \mathrm{New}$ outpatients.

United States. Information on caseload was available for two studies. Castel et al. [29] reported that pain treatment facilities in North Carolina had a high number $(\mathrm{M}=1244.10)$ of appointments per month. In 1985, Hickling et al. [31] reported new consultations $(\mathrm{M}=36.1)$ and follow-up appointments $(\mathrm{M}$ $=74.3$ ) to be significantly lower. No information was available regarding access to care or wait times.

3.4. Treatments Available at Pain Services. A wide range of medical, physical, psychological, and other treatments were reported at pain treatment facilities (see Tables 4-6).

Australia. Only one form of pain treatment was reported by Hogg et al. [20]. They reported that $3.5 \%$ of pain treatment facilities used acupuncture.
Canada. In three Canadian surveys $[18,21,22]$, medical treatments included cryotherapy (6\%), injections [trigger point injection (60-88\%), botulinic toxin injection (26-44\%), continuous epidural (80\%), one-shot epidural or epidural injection (46-100\%), facet joint/nerve injection (51-60\%), and intravenous regional anaesthesia (31-82\%)], nerve blocks [caudal block (37-74\%), paravertebral nerve block (40-48\%), peripheral nerve block (49-90\%), radiofrequency lesioning (40\%), stellate ganglion nerve block (42-92\%), and sympathetic block/local anesthetic (32-52\%)], pharmacotherapy (100\%), and spinal cord stimulation (12\%). Physical therapy related treatments included acupuncture (40-53\%), exercise programs (77-100\%), hydrotherapy (35-100\%), intramuscular stimulation (20-30\%), massage (20-34\%), physiotherapy 


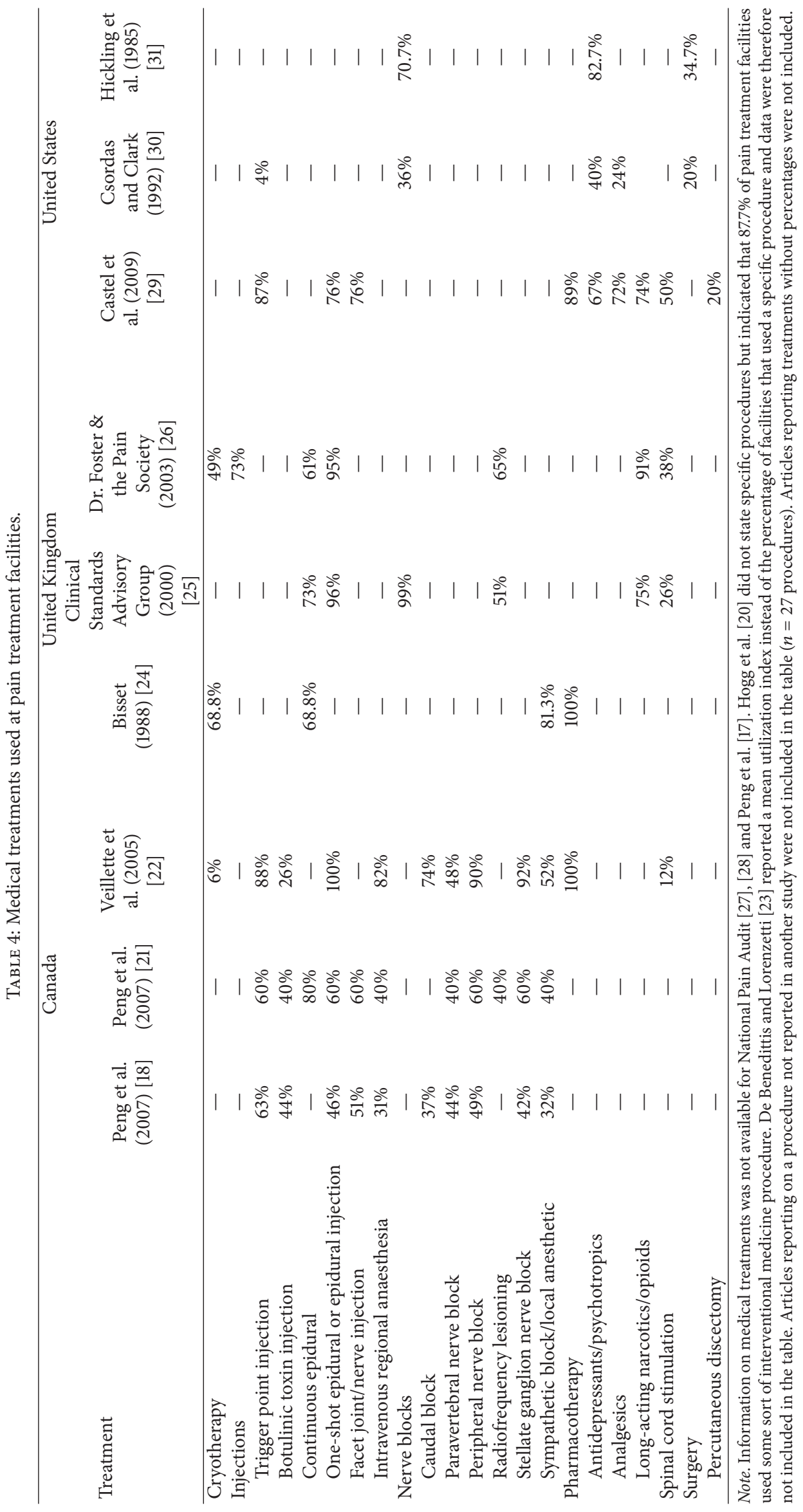




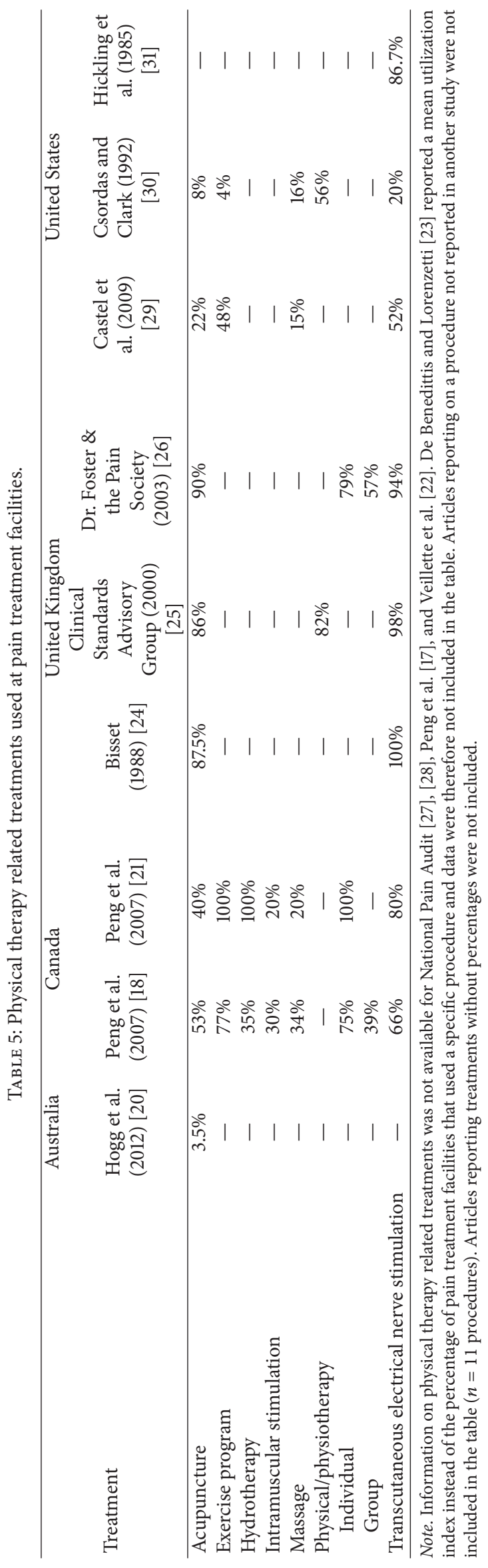


TABLE 6: Psychological treatments and other treatments used at pain treatment facilities.

\begin{tabular}{|c|c|c|c|c|c|c|c|c|}
\hline \multirow[b]{2}{*}{ Treatment } & \multicolumn{2}{|c|}{ Canada } & \multicolumn{3}{|c|}{ United Kingdom } & \multicolumn{3}{|c|}{ United States } \\
\hline & $\begin{array}{l}\text { Peng et al. } \\
(2007) \text { [18] }\end{array}$ & $\begin{array}{l}\text { Peng et al. } \\
\text { (2007) [21] }\end{array}$ & $\begin{array}{c}\text { Bisset } \\
\text { (1988) [24] }\end{array}$ & $\begin{array}{c}\text { Clinical } \\
\text { Standards } \\
\text { Advisory } \\
\text { Group (2000) } \\
{[25]}\end{array}$ & $\begin{array}{l}\text { Dr. Foster } \\
\text { \& the Pain } \\
\text { Society } \\
(2003) \\
{[26]}\end{array}$ & $\begin{array}{c}\text { Castel et } \\
\text { al. (2009) } \\
{[29]}\end{array}$ & $\begin{array}{c}\text { Csordas } \\
\text { and Clark } \\
(1992)[30]\end{array}$ & $\begin{array}{c}\text { Hickling et } \\
\text { al. (1985) } \\
{[31]}\end{array}$ \\
\hline \multicolumn{9}{|l|}{ Psychological treatments } \\
\hline Biofeedback & $39 \%$ & $80 \%$ & - & - & - & - & $48 \%$ & $85.3 \%$ \\
\hline Couple therapy & $25 \%^{\mathrm{a}}$ & - & - & - & - & - & - & $72 \%$ \\
\hline Education & - & - & - & - & - & $22 \%$ & $24 \%$ & - \\
\hline Family therapy & $25 \%^{\mathrm{a}}$ & $60 \%$ & - & - & - & - & - & $68 \%$ \\
\hline Group psychotherapy & $42 \%$ & $20 \%$ & - & - & - & - & - & - \\
\hline Hypnosis & $28 \%$ & $80 \%$ & $6.3 \%$ & $20 \%$ & - & - & $20 \%$ & - \\
\hline Imagery & $50 \%$ & $100 \%$ & - & - & - & - & - & - \\
\hline Individual psychotherapy & $79 \%$ & $100 \%$ & - & $66 \%$ & $69 \%$ & $22 \%$ & $80 \%$ & $92 \%$ \\
\hline Relaxation/breathing & $71 \%$ & $100 \%$ & - & - & - & - & $48 \%$ & - \\
\hline Support group & $42 \%$ & $40 \%$ & - & - & - & - & - & - \\
\hline \multicolumn{9}{|l|}{ Other treatments } \\
\hline Dietary/nutrition counseling & $47 \%$ & $40 \%$ & - & - & - & $35 \%$ & $12 \%$ & - \\
\hline Homeopathy & - & - & - & $4.5 \%$ & - & $20 \%$ & - & - \\
\hline Pharmaceutical counseling & $37 \%$ & $40 \%$ & - & - & - & - & - & - \\
\hline
\end{tabular}

Note. Information on psychological and other treatments was not available for Hogg et al. [20], National Pain Audit [27], [28], Peng et al. [17], and Veillette et al. [22]. De Benedittis and Lorenzetti [23] reported a mean utilization index instead of the percentage of pain treatment facilities that used a specific procedure and data were therefore not included in the table. Articles reporting on a procedure not reported in another study were not included in the table $(n=17$ procedures). Articles reporting treatments without percentages were not included.

${ }^{a}$ Reported as family/couple therapy.

(39-100\%), and transcutaneous electrical nerve stimulation $(66-80 \%)$. Psychological treatments included biofeedback (39-80\%), couples therapy (25\%), family therapy (25$60 \%)$, group psychotherapy (20-42\%), hypnosis $(28-80 \%)$, imagery (50-100\%), individual psychotherapy (79-100\%), relaxation/breathing (71-100\%), and support groups (40$42 \%)$. Other treatments included dietary/nutrition counseling (40-47\%) and pharmaceutical counseling (37-40\%).

Italy. De Benedittis and Lorenzetti [23] reported a mean utilization index instead of the percentage of facilities that used specific treatments and data was therefore not included in the tables. Of treatments that were reported in at least one other study, they reported that pain treatment facilities used nerve blocks, percutaneous discectomies, pharmacotherapy (including psychotropic medication, long-acting narcotics/opioids, and other medications), and spinal cord stimulation.

United Kingdom. Three surveys in the United Kingdom reported on treatments used in pain treatment facilities [2426]. Medical treatments included cryotherapy (49-68.8\%), injections [73\%: continuous epidural (61-73\%) and oneshot epidural or epidural injections (95-96\%)], nerve blocks [99\%: radiofrequency lesioning (51-65\%) and sympathetic block/local anesthetic (81.3\%)], long-acting narcotics/opioid medications (75-91\%), and spinal cord stimulation (26-38\%). Physical therapy related treatments included acupuncture
(87.5-90\%), physiotherapy (57-82\%), and transcutaneous electrical nerve stimulation (94-100\%). Psychological treatments included hypnosis (6.3-20\%) and individual psychotherapy (66-69\%). Other treatments included homeopathy $(4.5 \%)$.

United States. Medical treatments included injections [trigger point injections (4-87\%), one-shot epidural or epidural injections (76\%), and facet joint/nerve injections (76\%)], nerve blocks [caudal block (36-70.7\%)], pharmacotherapy [89\%: antidepressants/psychotropics (40-82.7\%), analgesics (24-74\%), and long-acting narcotics/opioids (74\%)], surgery (20-34.7\%), and percutaneous discectomies (20\%). Physical therapy related treatments included acupuncture (8-22\%), exercise programs (4-48\%), massage (15-16\%), physiotherapy (56\%), and transcutaneous electrical nerve stimulation (20$86.7 \%$ ). Psychological treatments included biofeedback (48$85.3 \%)$, couples therapy $(72 \%)$, education $(22-24 \%)$, family therapy (68\%), hypnosis (20\%), individual psychotherapy (22-92\%), and relaxation/breathing (48\%). Other treatments included dietary/nutrition counseling (12-35\%) and homeopathy (20\%).

3.5. Attributes of Pain Treatment Facilities. Data on the attributes of pain treatment facilities, including staff composition, space and equipment available, administrative support, and multidisciplinary status, are presented in Tables 7 and 8. 


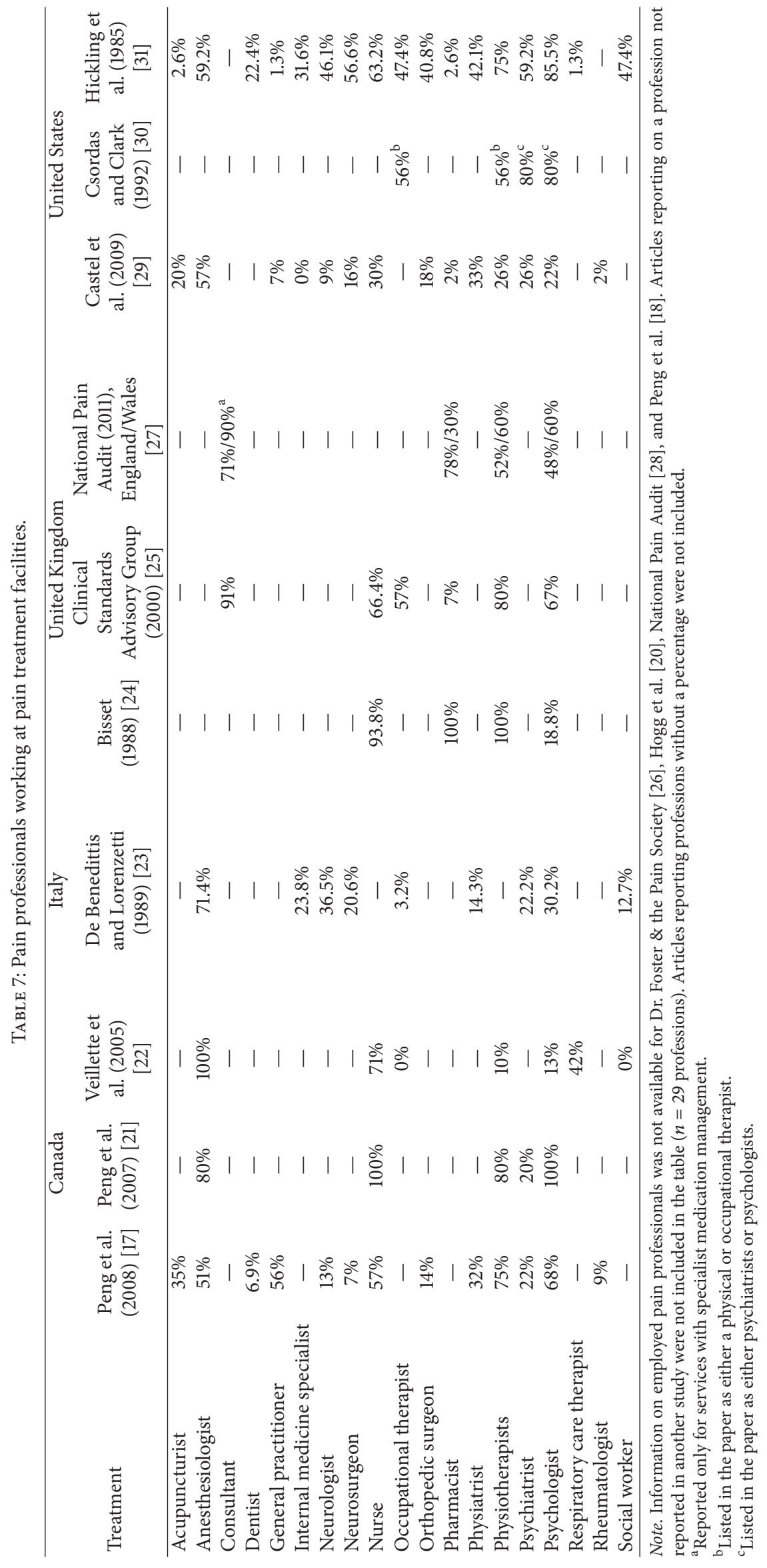




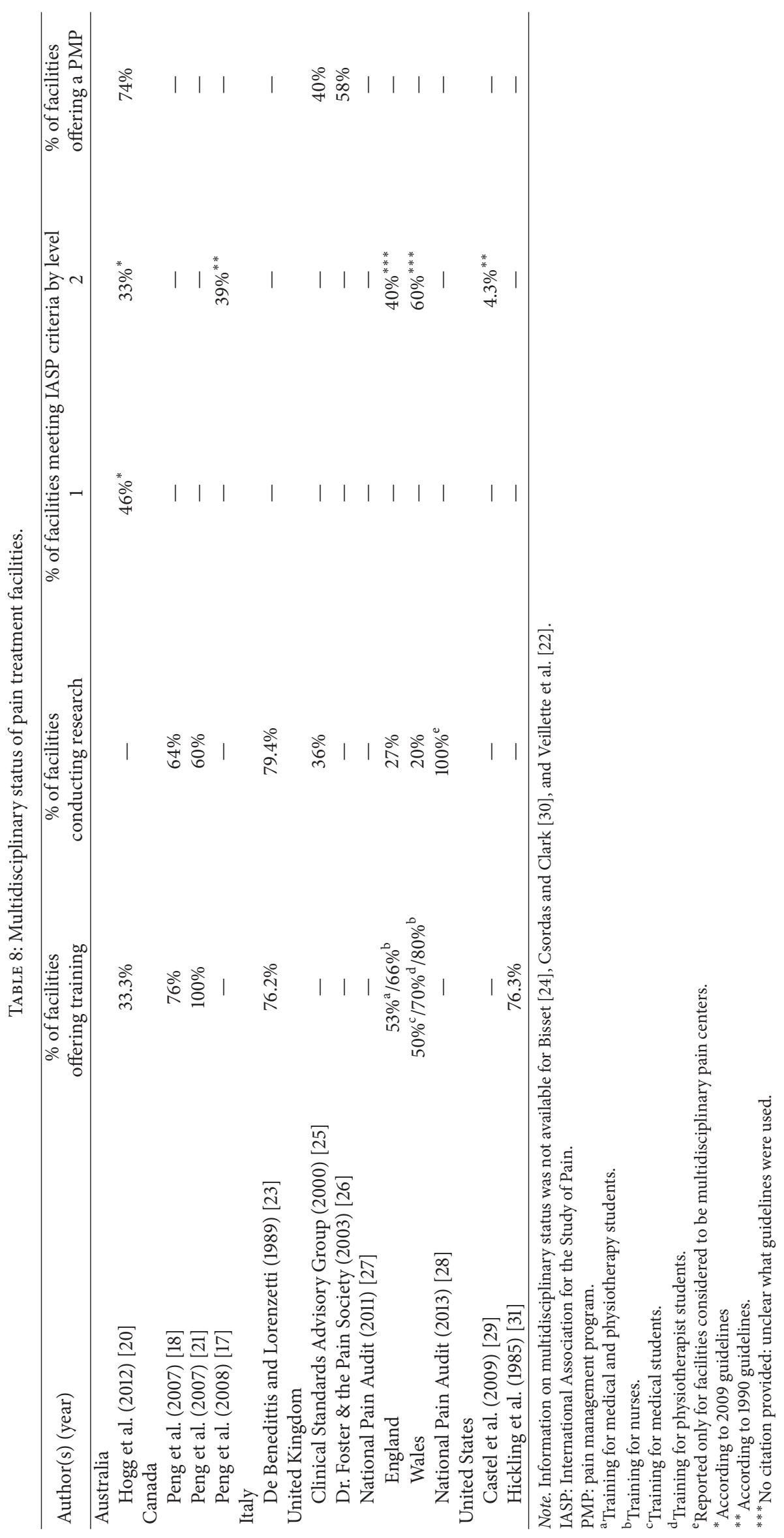


Australia. Information on pain professionals working at pain treatment facilities was not reported by Hogg et al. [20], although information on space and multidisciplinary status was available. It was reported that $60 \%$ of pain treatment facilities had inpatient care/beds [20]. The authors reported that $46 \%$ of pain facilities met Level 1 IASP criteria [14] (i.e., a multidisciplinary pain center) and 33\% met Level 2 criteria (i.e., a multidisciplinary pain clinic). Additionally, $33.3 \%$ of facilities offered training and $74 \%$ offered a pain management program (PMP) [20].

Canada. Three Canadian studies reported on the most frequently reported pain professionals working at pain treatment facilities $[17,21,22]$ : anesthesiologists (51-100\%), nurses (57-100\%), physiotherapists (10-80\%), and psychologists (13$100 \%)$. Two studies $[17,21]$ reported on psychiatrists $(20-$ $22 \%)$. Other reported pain professionals included acupuncturists, dentists, general practitioners, neurologists, neurosurgeons, occupational therapists, orthopedic surgeons, physiatrists, respiratory care therapists, rheumatologists, and social workers. Data on space, equipment, and staff at pain treatment facilities was only reported by Veillette et al. [22]. They reported that $52 \%$ had consultation/treatment room(s), 93.8\% had an operating theatre, $48 \%$ had recovery rooms, $75 \%$ had hospital/outpatient beds, and $77.6 \%$ had fluoroscopy equipment. Peng et al. [21] reported that $76 \%$ of adult pain treatment facilities offered training and $64 \%$ conducted research, with 39\% meeting IASP Level 2 criteria for multidisciplinary status (i.e., as multidisciplinary pain clinics, as reported by Peng et al. [17]). Peng et al. [18] reported that $100 \%$ of pediatric pain treatment facilities offered training and $60 \%$ conducted research.

Italy. De Benedittis and Lorenzetti [23] reported that the following health care professions were employed: anesthesiologists $(71.4 \%)$, internal medicine specialists $(23.8 \%)$, neurologists $(36.5 \%)$, neurosurgeons $(20.6 \%)$, occupational therapists (3.2\%), physiatrists $(14.3 \%)$, psychiatrists $(22.2 \%)$, psychologists (30.2\%), and social workers (12.7\%). The majority of pain treatment facilities reported having an outpatient clinic $(80.9 \%)$, a nerve block room $(57.1 \%)$, and an operating theatre $(50.8 \%)$. Inpatient care was reported in $41.3 \%$ and a day hospital in $44.4 \%$. Multidisciplinary status of pain treatment facilities was not assessed, although it was reported that $76.2 \%$ of facilities offered training and $79.4 \%$ conducted research.

United Kingdom. Three studies reported on the staffing of pain professionals [24, 25, 27]. The most frequently reported were pharmacists $(7-100 \%)$, physiotherapists (52$100 \%)$, psychologists (18.8-67\%), consultants (71-91\%), and nurses (66.4-93.8\%). Data on space, equipment, and staff were variable. The most commonly reported attribute was access to inpatient care (24-93.8\%). Other space, access, and equipment data reported included the availability of consultation rooms $(79 \%)$, operating theatres (81\%), hospital/outpatient beds (87.5\%), office space for administrative staff (80.3-83\%), office space for professional staff (66$84 \%)$, wheelchair access (80-100\%), and X-ray imaging (8993.8\%). The National Pain Audit [27] was the only survey published in the United Kingdom that reported data on the multidisciplinary status of pain treatment facilities, finding that $40 \%$ of English and $60 \%$ of Welsh facilities met IASP Level 2 criteria (i.e., as multidisciplinary pain clinics). Pain management programs were offered by $40 \%$ [25] and $58 \%$ [26]. The Clinical Standards Advisory Group [25] reported that $36 \%$ of facilities conducted research and $100 \%$ of multidisciplinary pain centers surveyed in the National Pain Audit [28] conducted research. National Pain Audit [27] reported that clinical training was provided to medical students, physiotherapy students, and nurses (range: 53-80\%) and that research was conducted at $27 \%$ of English facilities and $20 \%$ of Welsh facilities.

United States. Three surveys [29-31] reported on the incidence of employed physiotherapists (26-75\%), psychiatrists (26-80\%), and psychologists (22-85.5\%). Two surveys [2931] reported on the incidence of acupuncturists (2.6-50\%), anesthesiologists (57-59.2\%), general practitioners (1.3-7\%), internal medicine specialists $(0-31.6 \%)$, neurologists (9$46.1 \%)$, neurosurgeons (16-56.6\%), nurses (30-63.2\%), occupational therapists $(47.4-56 \%)$, orthopedic surgeons (18$40.8 \%$ ), pharmacists (2-2.6\%), and physiatrists (33-42.1\%). One survey reported on the employment of dentists $[22.4 \%$ [31]], respiratory care therapists [1.3\% [31]], rheumatologists [2\% [29]], and social workers [47.4\% [31]]. No American surveys reported data on space, equipment, or support staff. One study by Castel et al. [29] reported on the multidisciplinary status of pain treatment facilities, finding that only 4.3\% met IASP Level 2 criteria (i.e., as multidisciplinary pain clinics), although Hickling et al. [31] reported that $76.3 \%$ of surveyed facilities offered clinical training. Other studies did not use an existing classification for multidisciplinary status. For example, Csordas and Clark [30] reported that 28\% of pain facilities were comprehensive, $20 \%$ were syndrome specific, $8 \%$ were modality specific, $36 \%$ were partial clinics, and $8 \%$ were combined with other departments.

\section{Discussion}

The present study systematically reviewed the literature on surveys of pain treatment facilities. Our search yielded 14 English language studies published between 1985 and 2013. Surveys were conducted in Australia, Canada, Italy, the United Kingdom, and the United States. The results reveal little consistency in the research design and questionnaires used to describe pain treatment facilities. Each study used different search and inclusion criteria for pain treatment facilities. The availability of pain treatment facilities was scarce even though the caseloads and wait times for facilities were generally high. A wide range of interventional medical, physical, and psychological pain treatments were available. Most studies reported findings on the percentage of practitioners in different health care professions employed. Taken together, these findings suggest that there is considerable 
variability in the availability, services, and professionals of multidisciplinary pain clinics.

Each survey used a different approach to identify pain treatment facilities (Table 1). In some cases, facilities were identified through contacting individuals with membership in a specific association (e.g., [24]), searching the Yellow Pages (e.g., [29]), consulting a published list of facilities (e.g., $[25,31])$, or using a combination of different strategies. When the search for pain treatment facilities is not comprehensive, there is a risk of underestimating the availability of pain treatment facilities in a region and of selection bias that may undermine the representativeness of sampled pain facility characteristics. For example, Veillette et al. [22] identified pain treatment facilities by contacting the heads of anesthesia departments, a strategy that may have omitted facilities that are not directed by individuals in that role. This suggests that caution must be taken when interpreting and generalizing findings.

Pain treatment facilities are both scarce and in high demand (Tables 2 and 3). Access to multidisciplinary care varied by country, with one per 310,000 people in Australia [20], one per 258,000 people in Canada [18], and one per 200,000-370,370 people in the United Kingdom [27]. With an estimated $37 \%$ of the world population suffering from chronic pain [1], this reflects poor availability of services for pain sufferers. The demand for multidisciplinary pain treatment facilities is reflected in the wait times.

In 2005, IASP [33] developed recommendations for wait times based on an international task force of experts. They suggest wait times for acute painful conditions to be immediate, most urgent pain to be within one week, urgent or semiurgent pain to be within one month, and routine or regular pain to be within eight weeks. In the present study, we found one article from the United Kingdom that reported on wait times according to the severity of painful conditions [25] that showed the median wait times to be two weeks for urgent care and 16 weeks for routine care, both substantially longer than the suggested wait times. Two surveys showed breakdown of wait times according to public and private facilities $[18,20]$. It was found that wait times were longer in public facilities in Australia ( $\mathrm{Mdn}=21.4$ weeks) and Canada (Mdn $=25.7)$ in comparison to private facilities (Australia: Mdn = 5.5, Canada: $M d n=2.1$, showing that those that can afford to pay out of pocket for medical services can receive multidisciplinary pain treatment faster. No information was available on wait times from surveys conducted in the United States, where public funding is mainly limited to individuals that are 65 years old and older, individuals with disabilities, and some individuals with low income, although there are substantial gaps in health care coverage for the general American population [34]. It is possible that, in the United States, there may be increased demand for private services that may increase wait times. Importantly, it appears that access to the multidisciplinary care may be faster in pediatric settings: in a survey of five pediatric pain treatment facilities, Peng et al. [21] reported the median wait time to be four weeks, although it was not specified whether these facilities were public or private. Taken together, as longer wait times are associated with deterioration of health and lowered quality of life [35], the present findings suggest an alarming global situation in which access to pain treatment facilities requires improvement.

Unsurprisingly, it was found that medical pain treatments were reported more frequently in pain treatment facilities in acute care hospitals in Québec [22] in comparison to pain treatment facilities not restricted to hospital settings that were surveyed across Canada [18, 21]. The emphasis on medical treatments by Veillette et al. [22] is also illustrated in the exclusion of data collected on the prevalence of physical therapy and psychological and other treatments that were reported in Canada-wide surveys [18, 21]. Surveys conducted in the United Kingdom were published between 1988 and 2003. Despite the range, the prevalence of some reported treatments did not vary greatly. For example, continuous epidural was reportedly used in $68.8 \%$ of facilities in Bisset [24], 73\% in Clinical Standards Advisory Group [25], and $61 \%$ in the report by Dr. Foster \& the Pain Society [26]. However, it is noteworthy that only treatments reported in at least two studies (regardless of country of origin) were included in the review. In the three surveys conducted in the United States, the prevalence of injections and nerve blocks was only reported in two studies, with more information reported on pharmacotherapy treatments. Little information was available on pain treatments for surveys conducted in Australia and Italy, limiting the discussion on their use of interventions in pain treatment facilities.

According to IASP, multidisciplinary pain centers and clinics must be staffed by a variety of health care professionals that include physicians, nurses, mental health professionals, and physical therapists [14]. All Canadian surveys reported on the prevalence of these employed professionals, even though they were not always represented. However, in pediatric settings, Peng et al. [21] found that $100 \%$ of pain treatment facilities staffed a psychologist and a nurse, while $80 \%$ staffed an anaesthesiologist and physiotherapist. In Italy, the majority of pain facilities surveyed by De Benedittis and Lorenzetti [23] did not satisfy the staff requirements of a multidisciplinary pain centre or clinic given that physical therapists were reported in only $14.3 \%$ of facilities and mental health professionals were reported in $30.2 \%$. Data on the staff composition from surveys in the United Kingdom did not include any information on employed physicians, although the authors reported that nurses were staffed in up to $93.8 \%$ of facilities [24], physiotherapists in up to $100 \%$ [24], and psychologists in up to $67 \%$ [25]. In surveys conducted in the United States, Castel et al. [29] found that only a minority of facilities met IASP criteria for a multidisciplinary clinic and this low number was attributed to only having one provider type on staff. This may be related to the methodology used in this survey, as only pain facilities that specialized in treating neck and back pain were contacted for the survey. Although the staff composition was not surveyed by Hogg et al. [20], they reported that $46 \%$ of pain treatment facilities in Australia met IASP criteria for a multidisciplinary pain centre. Overall, even though many studies did not include a measure of the multidisciplinary functionality, an assessment of facilities provided demonstrates that pain treatment facilities attempt 
TABLE 9: Useful but infrequently reported survey questions.

\begin{tabular}{lc}
\hline Variable in questionnaire & $\begin{array}{c}\text { Number of articles } \\
\text { reporting on it }\end{array}$ \\
\hline Average cost of sessions, common treatments & 3 \\
Average length of the first appointment & 2 \\
Multidisciplinary pain facility ownership & 2 \\
Guidelines used & 1 \\
Inclusion criteria for referrals & 5 \\
Location of multidisciplinary pain facilities (e.g., in hospital, free-standing) & 2 \\
Major medical equipment available & 1 \\
Measure of data completeness and protocol for dealing with missing data & 2 \\
Measures of treatment effectiveness/outcome measures & 1 \\
Number of providers at each facility & 2 \\
Percentage of facilities that are urban or rural & 3 \\
Percentage of facilities that are public or private & 4 \\
Percentage of facilities treating children & 3 \\
Source of public and/or private funding & 3 \\
\hline
\end{tabular}

to provide comprehensive treatment to target both biomedical and psychological dimensions of pain.

This study has several limitations. The data extracted from the studies included in the review were heterogeneous, with surveys completed over a 28 -year period and in five countries. There are considerable differences in what is considered to be a pain treatment facility and the importance of various treatment strategies has changed over time [14, 36]. The method of identifying pain treatment facilities and the differing eligibility criteria introduced bias where not all multidisciplinary pain clinics may have been included in the reported surveys. This may have been similarly affected by the responses rate, which ranged between 56 and $100 \%$. Additionally, drawing inferences across the extracted data sample was challenging due to the extent of missing data and differently reported metrics.

Regular, comprehensive surveys and audits of pain treatment facilities are warranted. They provide important information on the availability of services, wait times to access treatment, and the types of services available. There were also many variables of interest for future research that were assessed in some studies but not others (see Table 9). These include information regarding the cost of treatments, outcome measures used, whether facilities are public or private, and guidelines that are currently in use. Future investigations should consider these variables. The Canadian Pain Coalition conducted this study as a part of the first phase of the Report Card on Pain. For the second phase, the Report Card on Pain aims to complete an updated survey of pain treatment facilities in Canada, as the most recent survey completed was over 8 years ago [18].

In summary, this systematic review of studies surveying pain treatment facilities around the world highlights important information for policymakers on the scope, demand, and accessibility of such facilities and provides direction for future researchers to enhance the comprehensiveness and comparability of data capture. The results showed that there is a great deal of diversity in the information collected and future studies should consider using more comprehensive search strategies, increasing clarity on what is considered to be a multidisciplinary pain treatment facility, and reporting on a consistent set of variables to provide a clear summary of the status of multidisciplinary pain treatment facilities. Despite their variability, the identified studies shed light on substantial gaps in tackling the challenge of pain and underscore the pressing need for improved access to pain treatment facilities globally.

\section{Summary}

This study systematically reviewed the published literature describing multidisciplinary chronic pain treatment facilities to provide an overview of their availability and characteristics. Fourteen articles satisfied inclusion criteria (i.e., original research that directly surveyed a range of treatments in more than one pain treatment facility). Results show considerable variability in the research methodologies used to describe pain treatment facilities, show that availability of pain treatment facilities was scarce, and show that accompanying caseloads and wait times were generally high. Pain treatment facilities used a wide range of pain interventions and employed a variety of health care professionals. Although the present study was limited by the heterogeneous data extracted from included studies, it highlights important information on the scope, demand, and accessibility of multidisciplinary pain treatment facilities.

\section{Appendices}

\section{A. Search Strategy for MEDLINE (OVID)}

(1) Pain/ 
(2) Pain.mp. [mp = title, abstract, original title, name of substance word, subject heading word, keyword heading word, protocol supplementary concept word, rare disease supplementary concept word, unique identifier]

(3) Pain clinics/

(4) Pain clinic ${ }^{*} . \mathrm{mp}$. [mp = title, abstract, original title, name of substance word, subject heading word, keyword heading word, protocol supplementary concept word, rare disease supplementary concept word, unique identifier]

(5) Pain service* .mp. [mp = title, abstract, original title, name of substance word, subject heading word, keyword heading word, protocol supplementary concept word, rare disease supplementary concept word, unique identifier]

(6) Pain cent ${ }^{*} . \mathrm{mp}$. [mp = title, abstract, original title, name of substance word, subject heading word, keyword heading word, protocol supplementary concept word, rare disease supplementary concept word, unique identifier]

(7) Pain facilit ${ }^{*} . m p$. [mp = title, abstract, original title, name of substance word, subject heading word, keyword heading word, protocol supplementary concept word, rare disease supplementary concept word, unique identifier]

(8) Pain management clinic ${ }^{*} . m p$. [mp $=$ title, abstract, original title, name of substance word, subject heading word, keyword heading word, protocol supplementary concept word, rare disease supplementary concept word, unique identifier]

(9) Pain management service*.$m p$. [mp $=$ title, abstract, original title, name of substance word, subject heading word, keyword heading word, protocol supplementary concept word, rare disease supplementary concept word, unique identifier]

(10) Pain management cent ${ }^{*} \cdot \mathrm{mp}$. [mp = title, abstract, original title, name of substance word, subject heading word, keyword heading word, protocol supplementary concept word, rare disease supplementary concept word, unique identifier]

(11) Pain management facility ${ }^{*} \cdot m p$. [mp $=$ title, abstract, original title, name of substance word, subject heading word, keyword heading word, protocol supplementary concept word, rare disease supplementary concept word, unique identifier]

(12) Pain treatment clinic ${ }^{*} . \mathrm{mp}$. [mp = title, abstract, original title, name of substance word, subject heading word, keyword heading word, protocol supplementary concept word, rare disease supplementary concept word, unique identifier]

(13) Pain treatment service*.mp. [mp = title, abstract, original title, name of substance word, subject heading word, keyword heading word, protocol supplementary concept word, rare disease supplementary concept word, unique identifier]
(14) Pain treatment cent ${ }^{*} . \mathrm{mp}$. [mp = title, abstract, original title, name of substance word, subject heading word, keyword heading word, protocol supplementary concept word, rare disease supplementary concept word, unique identifier]

(15) Pain treatment facilit ${ }^{*} . \mathrm{mp}$. [mp = title, abstract, original title, name of substance word, subject heading word, keyword heading word, protocol supplementary concept word, rare disease supplementary concept word, unique identifier]

(16) Health surveys/or health care surveys/

(17) Survey* ${ }^{*} . \mathrm{mp}$. [mp = title, abstract, original title, name of substance word, subject heading word, keyword heading word, protocol supplementary concept word, rare disease supplementary concept word, unique identifier]

(18) Program evaluation/

(19) Program evaluation.mp. [ $\mathrm{mp}=$ title, abstract, original title, name of substance word, subject heading word, keyword heading word, protocol supplementary concept word, rare disease supplementary concept word, unique identifier]

(20) Questionnaires/

(21) Questionnaire*.mp. [mp = title, abstract, original title, name of substance word, subject heading word, keyword heading word, protocol supplementary concept word, rare disease supplementary concept word, unique identifier]

(22) Program assessment.mp. [mp = title, abstract, original title, name of substance word, subject heading word, keyword heading word, protocol supplementary concept word, rare disease supplementary concept word, unique identifier]

(23) Program outcome.mp. [ $\mathrm{mp}=$ title, abstract, original title, name of substance word, subject heading word, keyword heading word, protocol supplementary concept word, rare disease supplementary concept word, unique identifier]

(24) \#1 or \#2

(25) \#3 or \#4 or \#5 or \#6 or \#7 or \#8 or \#9 or \#10 or \#11 or $\# 12$ or $\# 13$ or \#14 or \#15

(26) \#16 or \#17 or \#18 or \#19 or \#20 or \#21 or \#22 or \#23

(27) \#24 or \#25

(28) \#26 or \#27

\section{B. Search Strategy for CINAHL and PsycINFO (ProQuest)}

ALL (pain) AND ALL ("pain clinic*” OR "pain service*” OR "pain cent" OR "pain facilit*” OR "pain management clinic*” OR "pain management service*” OR "pain management cent" ${ }^{*}$ OR "pain management facilit" treatment clinic"” OR "pain treatment service*” OR "pain treatment cent" " OR "pain treatment facilit"”) AND ALL 
("survey*” OR "program evaluation" OR "program assessment" OR "program outcome" OR “questionnaire*”).

\section{Disclosure}

A portion of the research in this paper was presented as a poster and oral presentation at the Canadian Pain Society conference in May 2015. An abstract of the poster and presentation was published in Pain Research \& Management (vol. 20, issue 3, e28-e75, 2015).

\section{Conflict of Interests}

The authors declare no conflict of interests.

\section{Acknowledgments}

Samantha Fashler is supported by an Ontario Graduate Scholarship and a Canadian Institutes of Health Research (CIHR) Vanier Canada Graduate Scholarship. Lindsay Burns is supported by a Frederick Banting and Charles Best CIHR Doctoral Scholarship. Joel Katz is supported by a CIHR Canada Research Chair in Health Psychology. This project was conducted in collaboration with the Canadian Pain Coalition (CPC) as a part of the Report Card on Pain.

\section{References}

[1] A. Tsang, M. Von Korff, S. Lee et al., "Common chronic pain conditions in developed and developing countries: gender and age differences and comorbidity with depression-anxiety disorders," Journal of Pain, vol. 9, no. 10, pp. 883-891, 2008.

[2] A. Boulanger, A. J. Clark, P. Squire, E. Cui, and G. L. A. Horbay, "Chronic pain in Canada: have we improved our management of chronic noncancer pain?" Pain Research and Management, vol. 12, no. 1, pp. 39-47, 2007.

[3] D. A. Tripp, E. G. VanDenKerkhof, and M. McAlister, "Prevalence and determinants of pain and pain-related disability in urban and rural settings in southeastern Ontario," Pain Research and Management, vol. 11, no. 4, pp. 225-233, 2006.

[4] L. R. Miller and A. Cano, "Comorbid chronic pain and depression: who is at risk?" Journal of Pain, vol. 10, no. 6, pp. 619-627, 2009.

[5] G. J. G. Asmundson and J. Katz, "Understanding the cooccurrence of anxiety disorders and chronic pain: state-of-theart," Depression and Anxiety, vol. 26, no. 10, pp. 888-901, 2009.

[6] K. S. Dillie, M. F. Fleming, M. P. Mundt, and M. T. French, "Quality of life associated with daily opioid therapy in a primary care chronic pain sample," Journal of the American Board of Family Medicine, vol. 21, no. 2, pp. 108-117, 2008.

[7] D. J. Gaskin and P. Richard, "The economic costs of pain in the United States," Journal of Pain, vol. 13, no. 8, pp. 715-724, 2012.

[8] M. Choinière, D. Dion, P. Peng et al., "The Canadian STOPPAIN project-part 1: who are the patients on the waitlists of multidisciplinary pain treatment facilities?" Canadian Journal of Anesthesia, vol. 57, no. 6, pp. 539-548, 2010.

[9] C. Phillips and D. Schopflocher, "The economics of chronic pain," in Health Policy Perspectives on Chronic Pain, S. Rashiq, P. Taenzer, and D. Schopflocher, Eds., pp. 41-50, John Wiley \& Sons, Weinheim, Germany, 2008.
[10] R. J. Gatchel, Y. B. Peng, M. L. Peters, P. N. Fuchs, and D. C. Turk, "The biopsychosocial approach to chronic pain: scientific advances and future directions," Psychological Bulletin, vol. 133, no. 4, pp. 581-624, 2007.

[11] L. Scascighini, V. Toma, S. Dober-Spielmann, and H. Sprott, "Multidisciplinary treatment for chronic pain: a systematic review of interventions and outcomes," Rheumatology, vol. 47, no. 5, pp. 670-678, 2008.

[12] R. J. Gatchel and A. Okifuji, "Evidence-based scientific data documenting the treatment and cost-effectiveness of comprehensive pain programs for chronic nonmalignant pain," Journal of Pain, vol. 7, no. 11, pp. 779-793, 2006.

[13] M. Ospina and C. Harstall, Multidisciplinary Pain Programs for Chronic Pain: Evidence from Systematic Reviews, Health Technology Assessment, Alberta Heritage Foundation for Medical Research, Edmonton, Canada, 2003.

[14] International Association for the Study of Pain, "Recommendations for pain treatment services 2009," http://www. iasp-pain.org/Education/Content.aspx?ItemNumber=1381.

[15] International Association for the Study of Pain. Desirable characteristics for pain treatment facilities 1990, http://www.iasppain.org/education/content.aspx?itemnumber $=1471$.

[16] A. Liberati, D. G. Altman, J. Tetzlaff et al., "The PRISMA statement for reporting systematic reviews and meta-analyses of studies that evaluate health care interventions: explanation and elaboration," Annals of Internal Medicine, vol. 151, no. 4, pp. W-65-W-94, 2009.

[17] P. Peng, J. N. Stinson, M. Choiniere et al., "Role of health care professionals in multidisciplinary pain treatment facilities in Canada," Pain Research and Management, vol. 13, no. 6, pp. 484488, 2008.

[18] P. Peng, M. Choiniere, D. Dion et al., "Challenges in accessing multidisciplinary pain treatment facilities in Canada," Canadian Journal of Anesthesia, vol. 54, no. 12, pp. 977-984, 2007.

[19] J. Higgins and S. Green, "Cochrane handbook for systematic reviews of interventions version 5.0.0," 2008, http://www. cochrane-handbook.org/.

[20] M. N. Hogg, S. Gibson, A. Helou, J. Degabriele, and M. J. Farrell, "Waiting in pain: a systematic investigation into the provision of persistent pain services in Australia," Medical Journal of Australia, vol. 196, no. 6, pp. 386-390, 2012.

[21] P. Peng, J. N. Stinson, M. Choiniere et al., "Dedicated multidisciplinary pain management centres for children in Canada: the current status," Canadian Journal of Anesthesia, vol. 54, no. 12, pp. 985-991, 2007.

[22] Y. Veillette, D. Dion, N. Altier, and M. Choinière, "The treatment of chronic pain in Québec: a study of hospital-based services offered within anesthesia departments," Canadian Journal of Anesthesia, vol. 52, no. 6, pp. 600-606, 2005.

[23] G. De Benedittis and A. Lorenzetti, "The current status of pain clinics in Italy: a questionnaire survey," Clinical Journal of Pain, vol. 5, no. 3, pp. 261-269, 1989.

[24] W. I. K. Bisset, "Pain relief clinics under anaesthetic management in Scotland," Annals of the Royal College of Surgeons of England, vol. 70, no. 6, pp. 392-394, 1988.

[25] Clinical Standards Advisory Group, Services for Patients with Pain, Department of Health, London, UK, 2000.

[26] Dr Foster \& the Pain Society, Adult Chronic Pain Management Services in the UK, British Pain Society Publications, London, UK, 2003. 
[27] National Pain Audit, Phase 1 Report: Organisational Audit of NHS Chronic Pain Services, Dr Foster Research, London, UK, 2011.

[28] National Pain Audit, Third Report: Focus on Safety and Outcomes, Dr Foster Research, London, UK, 2013.

[29] L. D. Castel, J. K. Freburger, G. M. Holmes, R. P. Scheinman, A. M. Jackman, and T. S. Carey, "Spine and pain clinics serving North Carolina patients with back and neck pain: what do they do, and are they multidisciplinary?" Spine, vol. 34, no. 6, pp. 615622, 2009.

[30] T. J. Csordas and J. A. Clark, "Ends of the line: diversity among chronic pain centers," Social Science \& Medicine, vol. 34, no. 4, pp. 383-393, 1992.

[31] E. J. Hickling, G. F. P. Sison Jr., and J. L. Holtz, "Role of psychologists in multidisciplinary pain clinics: a national survey," Professional Psychology: Research and Practice, vol. 16, no. 6, pp. 868-880, 1985.

[32] World Health Organization, Health Systems, World Health Organization, Geneva, Switzerland, 2015, http://www.who.int/ topics/health_systems/en/.

[33] International Association for the Study of Pain, "Recommendations for wait-times for treatment of pain: summary and recommendations," 2005, https://www.iasp-pain.org/files/ Content/NavigationMenu/EducationalResources/IASP_Wait Times.pdf.

[34] E. Mossialos, M. Wenzl, R. Osborn, and C. Anderson, International Profiles of Health Care Systems, 2014, The Commonwealth Fund, New York, NY, USA, 2015.

[35] M. E. Lynch, F. Campbell, A. J. Clark et al., "A systematic review of the effect of waiting for treatment for chronic pain," Pain, vol. 136, no. 1-2, pp. 97-116, 2008.

[36] M. A. Gardea and R. J. Gatchel, "Interdisciplinary treatment of chronic pain," Current Review of Pain, vol. 4, no. 1, pp. 18-23, 2000. 


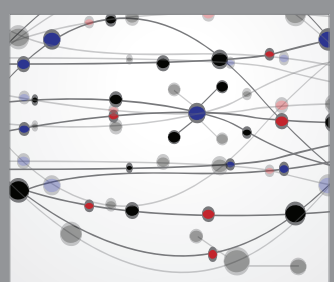

The Scientific World Journal
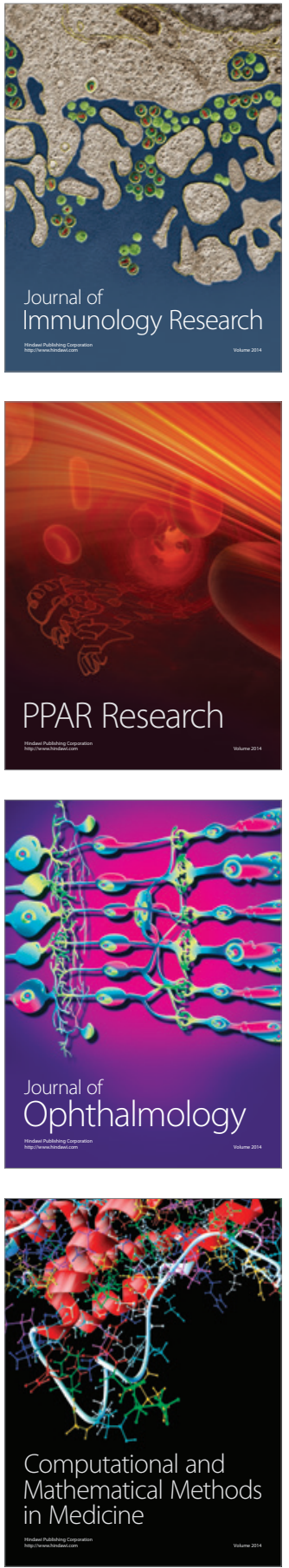

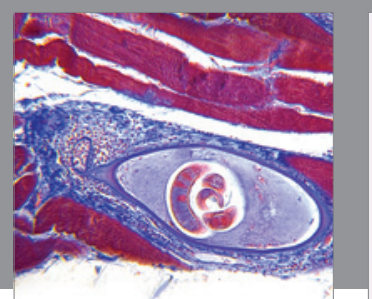

Gastroenterology Research and Practice

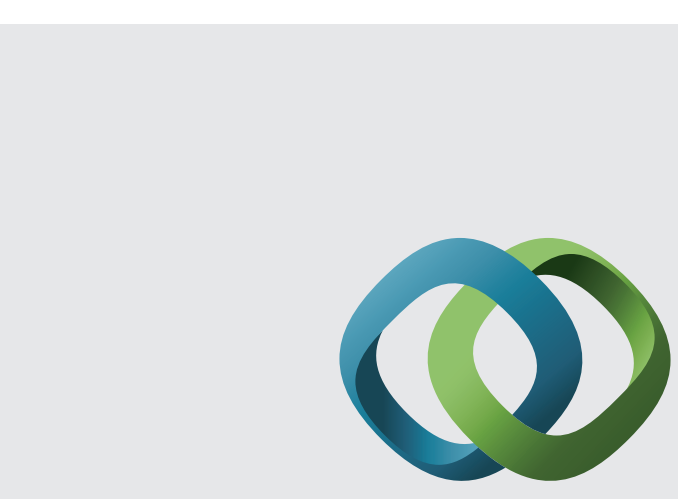

\section{Hindawi}

Submit your manuscripts at

http://www.hindawi.com
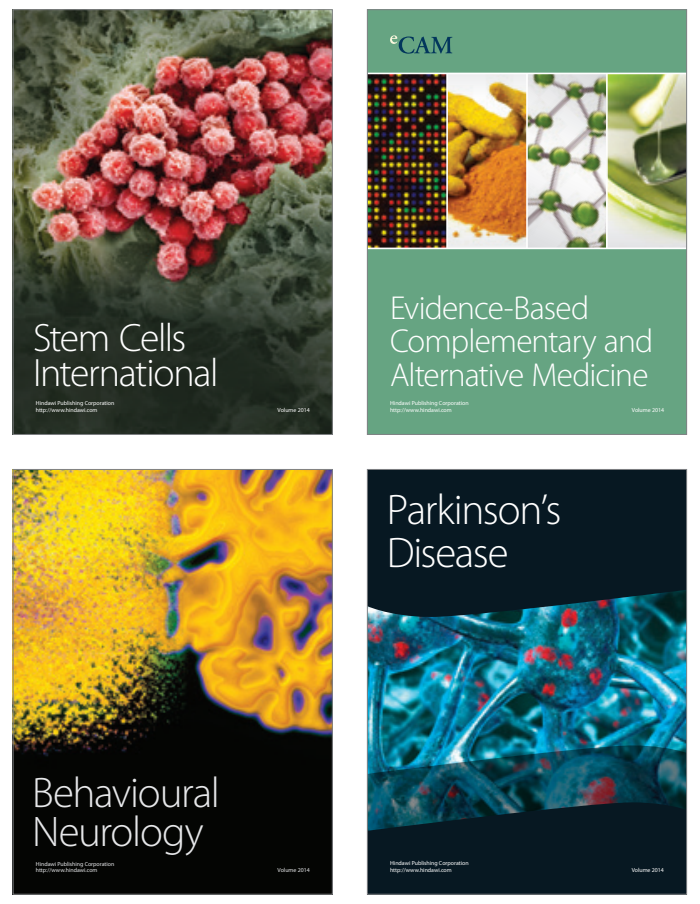
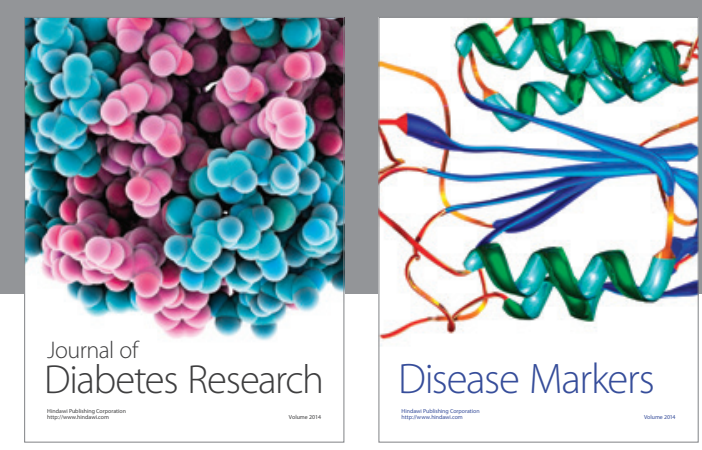

Disease Markers
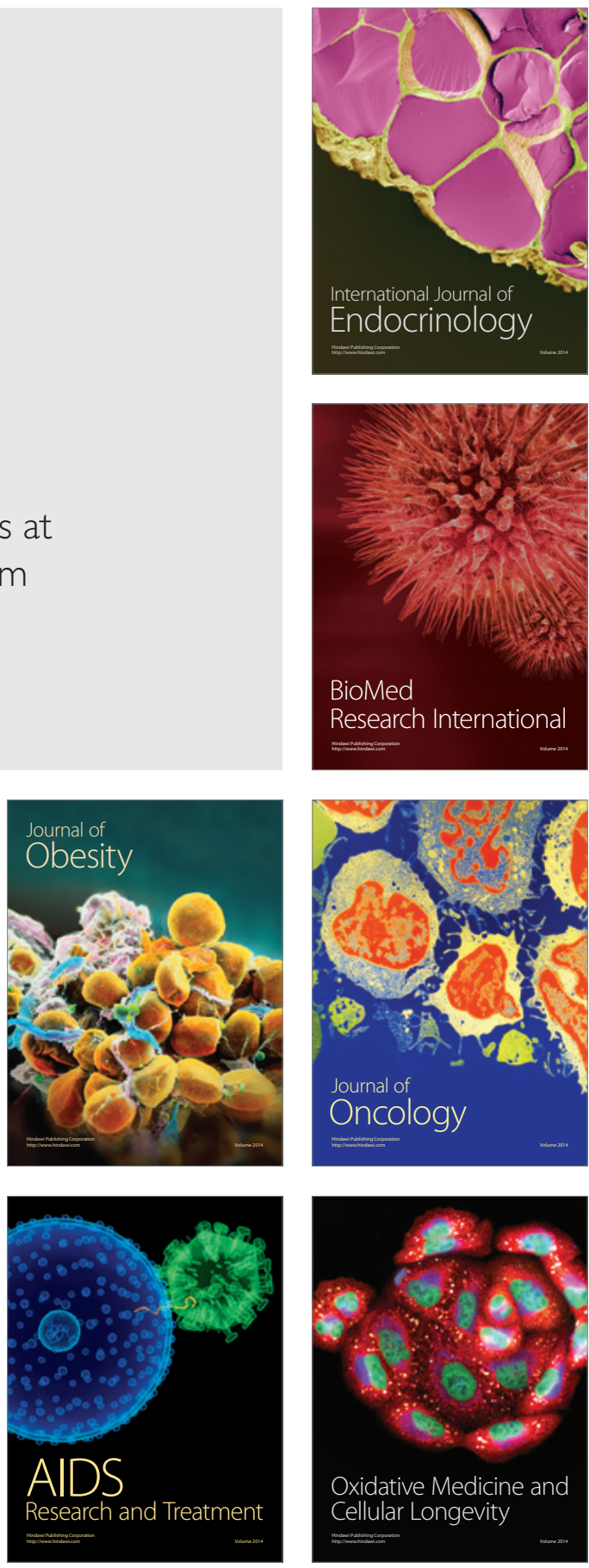